\title{
The Calculation for Strain Distributions and Electronic Structure of InAs/GaAs Quantum Dots Based on the Eight-Band $\boldsymbol{k} \cdot \boldsymbol{p}$ Theory
}

\author{
ChangGan Shu ${ }^{a, *}$ And Yumin LiU ${ }^{a, b}$ \\ ${ }^{a}$ State Key Laboratory of IPOC, Beijing University of Posts and Telecommunications, Beijing 100876, China \\ ${ }^{b}$ Institute of Semiconductors, Chinese Academy of Sciences, Beijing 100083, China
}

(Received September 9, 2015)

\begin{abstract}
In order to analyze the strain distribution of InAs/GaAs quantum dot in a pyramidal geometry, the traditional calculation method is based on the single band envelope approximation with the modified band edge from the eight band $k \cdot p$ theory. In this paper, we use the eight band $k \cdot p$ Hamiltonian to calculate, and the piezoelectric effects and the electronic structure are also discussed subsequently. To this end, some necessarily derived formulae in calculations about using the finite element calculation software COMSOL are presented in this paper. The results show the details about strain distributions, piezoelectric effects and electronic structure of an InAs/GaAs pyramidal quantum dot, verify the feasibility and efficiency of the calculation method.
\end{abstract}

DOI: 10.12693/APhysPolA.129.371

PACS/topics: 73.21.La, 73.63.Kv

\section{Introduction}

Quantum dots (QDs) are tiny crystal structure at the nanoscale, having full localization wave functions and quantization spectroscopy. Three-dimensional confinements QD system has distinct energy state characteristics of electrons like atoms compared with traditional bulk materials, one-dimensional confinement quantum well materials, and two-dimensional confinements quantum wire materials [1-3], caused extensive concern in some research fields such as optoelectronic devices, microelectronic devices, and quantum electronics [4, 5]. Self-organized growth is the main route of QDs fabrication. Strain self-organized QDs have considerable application potentialities in the areas of light-emitting devices and quantum electronics, becoming a research hotspot.

Strain plays an important role in growing strain selforganized QDs. First, strain is the most important driving force in QDs self-organized growth. The more study in strain and stress, the better we understand about the self-organized principles. Second, the variation of strain distributions correlates fairly with the electronic structures of QDs, which affect the optoelectronic characteristic of QD devices [6,7].

There are a few ways to analyze the strain of QDs, which can be summarized to three methods: (a) The molecular dynamic method [8], the potential functions are used to calculate the interactions of atoms in this method. Different materials correspond to different potential functions models, taking the InAs/GaAs QD for example, the Stranski-Krastanow (S-K) growth mode is one of the most common approaches. However, this method is suitable to describe the small volume

\footnotetext{
*corresponding author; e-mail: bupt_scg@163.com
}

isolated QD systems. (b) Continuum elastic energy theory [9], assuming a QD is buried in an infinite or semiinfinite substrate material, the strain distributions of QD and materials can be changed into the QD surface Green function integral. The assumption used in this method influences the authenticity of results about the strain. (c) Numerical calculation method based on continuity and unity [10], assuming a definite shape QD, the minimum of strain energy is obtained using the finite element method (FEM) without other priori conditions.

In this paper, we focus on the strain distributions of the InAs/GaAs QD in a pyramidal geometry. We could find that, in order to obtain information about the strain, piezoelectric effects and electronic structure, the traditional calculation method is based on the single band envelope approximation with the modified band edge from the eight band $k \cdot p$ theory. However, in this paper, we use the eight band $k \cdot p$ Hamiltonian to calculate. In order to facilitate using the simulation software COMSOL to solve the eight complicated partial differential equations which are tightly coupled, we also present some necessary derived formulae to comprehend the calculation method effectively. Finally some discussions and conclusions are given.

\section{Strain calculations}

We use a stiffness matrix $D$ to set up the dependence of the strain components on stress. The strain and the stress can be expressed as

$$
\varepsilon=\left(\begin{array}{c}
\varepsilon_{x x} \\
\varepsilon_{y y} \\
\varepsilon_{z z} \\
\varepsilon_{x y} \\
\varepsilon_{y z} \\
\varepsilon_{z x}
\end{array}\right)=\left(\begin{array}{c}
\partial u / \partial x \\
\partial v / \partial y \\
\partial w / \partial z \\
(\partial u / \partial y+\partial v / \partial x) / 2 \\
(\partial v / \partial z+\partial w / \partial y) / 2 \\
(\partial u / \partial z+\partial w / \partial x) / 2
\end{array}\right),
$$


$\sigma=D\left(\varepsilon-\varepsilon_{0}\right)+\sigma_{0}$,

where the displacements along the three-dimensional coordinates are $u, v$, and $w$. $D$ is the stiffness matrix. The original state of stress is $\sigma_{0}$, which could be considered as zero across the entire system. The original state of strain is $\varepsilon_{0}$, which is the lattice mismatch of the heterostructure in the QD, represented as

$$
\varepsilon_{0}= \begin{cases}\varepsilon_{i i}=\left(a_{\mathrm{InAs}}-a_{\mathrm{GaAs}}\right) / a_{\mathrm{GaAs}}, & i=x, y, z, \\ \varepsilon_{i j}=0, & i \neq j .\end{cases}
$$

Consider a zinc-blende QD, the elastic constant tensor is represented as

$$
D=\left(\begin{array}{cccccc}
C_{11} & C_{12} & C_{12} & 0 & 0 & 0 \\
C_{12} & C_{11} & C_{12} & 0 & 0 & 0 \\
C_{12} & C_{12} & C_{11} & 0 & 0 & 0 \\
0 & 0 & 0 & C_{44} & 0 & 0 \\
0 & 0 & 0 & 0 & C_{44} & 0 \\
0 & 0 & 0 & 0 & 0 & C_{44}
\end{array}\right) .
$$

The linear continuum elastic theory is used to calculate the strain distributions. The FEM with periodic boundary conditions is used in this paper. The system elastic energy is minimized by the conjugate gradient algorithm which means the lattice structures tends to stability. Consider the InAs/GaAs QD as a square-based pyramid and the wetting layer could be ignored when calculating the strain [11].

\section{Piezoelectric effects}

Piezoelectric effect is an electric polarization phenomenon caused by stress which effect on the asymmetric crystal [12-14]. The strain tensor in this system can be calculated according to the minimum potential energy principle of elastic mechanics. We could obtain the linear polarization field $P$ according to the strain tensor or the stress tensor from the formula

$$
P_{i}=\sum_{l m} e_{i l m}(r) \varepsilon_{l m}(r) .
$$

The formula above can be written in detail

$$
\left(\begin{array}{c}
P_{x} \\
P_{y} \\
P_{z}
\end{array}\right)=\left(\begin{array}{llllll}
e_{11} & e_{12} & e_{13} & e_{14} & e_{15} & e_{16} \\
e_{21} & e_{22} & e_{23} & e_{24} & e_{25} & e_{26} \\
e_{31} & e_{32} & e_{33} & e_{34} & e_{35} & e_{36}
\end{array}\right)\left(\begin{array}{l}
\varepsilon_{x x} \\
\varepsilon_{y y} \\
\varepsilon_{z z} \\
2 \varepsilon_{y z} \\
2 \varepsilon_{z x} \\
2 \varepsilon_{x y}
\end{array}\right) .
$$

Some redundant information are included in the formula (6) and the necessary simplification is taken. For the zinc-blende InAs/GaAs material, $e_{14}=e_{25}=e_{36}$, other elements are zero, so the polarization $P$ can be represented as

$$
\left(\begin{array}{c}
P_{x} \\
P_{y} \\
P_{z}
\end{array}\right)=\left(\begin{array}{ccc}
e_{14} & 0 & 0 \\
0 & e_{14} & 0 \\
0 & 0 & e_{14}
\end{array}\right)\left(\begin{array}{c}
2 \varepsilon_{y z} \\
2 \varepsilon_{z x} \\
2 \varepsilon_{x y}
\end{array}\right) .
$$

Note that the second order polarization has become an important part of the total polarization. On the basis of density functional theory, the second order polarization of InAs/GaAs can be derived [15]:

$$
\begin{gathered}
P^{(2)}=2 B_{114}\left(\begin{array}{c}
\varepsilon_{x x} \varepsilon_{y y} \\
\varepsilon_{y y} \varepsilon_{x z} \\
\varepsilon_{z z} \varepsilon_{x y}
\end{array}\right)+2 B_{124}\left(\begin{array}{c}
\varepsilon_{y z}\left(\varepsilon_{y y}+\varepsilon_{z z}\right) \\
\varepsilon_{x z}\left(\varepsilon_{x x}+\varepsilon_{z z}\right) \\
\varepsilon_{x y}\left(\varepsilon_{x x}+\varepsilon_{y y}\right)
\end{array}\right) \\
+4 B_{156}\left(\begin{array}{c}
\varepsilon_{x z} \varepsilon_{x y} \\
\varepsilon_{y z} \varepsilon_{x y} \\
\varepsilon_{y z} \varepsilon_{x z}
\end{array}\right) .
\end{gathered}
$$

The values of $B_{i j k}$ we used in for calculation are given in Table I, unit is $C / m^{2}$.

The values of $B_{i j k}$.

TABLE I

\begin{tabular}{c|c|c|c}
\hline \hline & $B_{114}$ & $B_{124}$ & $B_{156}$ \\
\hline InAs & -0.531 & -4.076 & -0.120 \\
GaAs & -0.439 & -3.765 & -0.492
\end{tabular}

To the divergence operation of the total polarization, written as $\left(P+P^{(2)}\right)$, we get the fixed piezoelectric charge density

$$
\rho(r)=-\nabla \cdot\left(P+P^{(2)}\right) .
$$

In the light of the Poisson equation, the piezoelectric potential $V_{P}$ can be calculated.

$$
\varepsilon_{0} \nabla \cdot\left(\varepsilon_{r}(r) \nabla V_{P}\right)=\rho,
$$

where $\varepsilon_{0}$ is the permittivity of vacuum, $\varepsilon_{r}$ is the relative static dielectric tensor.

\section{Electronic structure calculations}

In this section, we use COMSOL software to investigate the electronic structure based on eight bands $k \cdot p$ Hamiltonian

$$
H=H_{0}+H_{\mathrm{s}}+V,
$$

where $H_{0}$ is the kinetic piece of the Hamiltonian, expressed as

$$
H_{0}=\left(\begin{array}{cccccccc}
A & 0 & V^{\dagger} & 0 & \sqrt{3} V & -\sqrt{2} U & -U & \sqrt{2} V^{\dagger} \\
0 & A & -\sqrt{2} U & -\sqrt{3} V^{\dagger} & 0 & -V & \sqrt{2} V & U \\
V & -\sqrt{2} U & -P+Q & -S^{\dagger} & R & 0 & \sqrt{\frac{3}{2}} S & -\sqrt{2} Q \\
0 & -\sqrt{3} V & -S & -P-Q & 0 & R & -\sqrt{2} R & \sqrt{\frac{1}{2}} S \\
\sqrt{3} V^{\dagger} & 0 & R^{+} & 0 & -P-Q & S^{\dagger} & \sqrt{\frac{1}{2}} S^{\dagger} & \sqrt{2} R^{\dagger} \\
-\sqrt{2} U & -V^{\dagger} & 0 & R^{+} & S & -P+Q & \sqrt{2} Q & \sqrt{\frac{3}{2}} S^{\dagger} \\
-U & \sqrt{2} V^{\dagger} & \sqrt{\frac{3}{2}} S^{\dagger} & -\sqrt{2} R^{\dagger} & \sqrt{\frac{1}{2}} S & \sqrt{2} Q & -P-\Delta & 0 \\
\sqrt{2} V & U & -\sqrt{2} Q & \sqrt{\frac{1}{2}} S^{\dagger} & \sqrt{2} R & \sqrt{\frac{3}{2}} S & 0 & -P-\Delta
\end{array}\right),
$$


where the superscript " $\nmid$ " denotes the complex conjugate transpose operation. Here the components of $H_{0}$ are given

$$
\left\{\begin{array}{l}
A=\frac{\hbar^{2}}{2 m_{0}}\left(k_{x}^{2}+k_{y}^{2}+k_{z}^{2}\right)+E_{\mathrm{c}}, \\
P=\frac{\hbar^{2}}{2 m_{0}} \gamma_{1}\left(k_{x}^{2}+k_{y}^{2}+k_{z}^{2}\right)-E_{\mathrm{v}}, \\
Q=\frac{\hbar^{2}}{2 m_{0}} \gamma_{2}\left(k_{x}^{2}+k_{y}^{2}-2 k_{z}^{2}\right), \\
R=\sqrt{3} \frac{\hbar^{2}}{2 m_{0}}\left[-\gamma_{2}\left(k_{x}^{2}-k_{y}^{2}\right)+2 \mathrm{i} \gamma_{3} k_{x} k_{y}\right], \\
S=\sqrt{3} \gamma_{3} \frac{\hbar^{2}}{m_{0}} k_{z}\left(k_{x}-\mathrm{i} k_{y}\right), \\
U=\frac{1}{\sqrt{3}} P_{0} k_{z}, \\
V=\frac{1}{\sqrt{6}} P_{0}\left(k_{x}-\mathrm{i} k_{y}\right) .
\end{array}\right.
$$

Some necessary explanations: $E_{\mathrm{c}}$ and $E_{\mathrm{v}}$ are the conduction band and valence band before strained. $V$ is the confining potential. $P$ and $Q$ are the changes of valence band edge caused by strain. $P_{0}$ denotes the band mixing of the two bands above.
$\Delta$ denotes the spin-orbit splitting. Some Luttinger parameters are defined as:

$$
\left\{\begin{array}{l}
\gamma_{1}=\gamma_{1}^{\mathrm{L}}-\frac{E_{p}}{3 E_{\mathrm{g}}+\Delta}, \\
\gamma_{2}=\gamma_{2}^{\mathrm{L}}-\frac{1}{2} \frac{E_{p}}{3 E_{\mathrm{g}}+\Delta}, \\
\gamma_{3}=\gamma_{3}^{\mathrm{L}}-\frac{1}{2} \frac{E_{p}}{3 E_{\mathrm{g}}+\Delta},
\end{array}\right.
$$

The values of $\gamma_{i}^{\mathrm{L}}$ we used for calculation are given in Table II, where the energy gap $E_{\mathrm{g}}=E_{\mathrm{c}}-E_{\mathrm{v}}$, and $E_{p}=2 m_{0} P_{0}^{2} / \hbar^{2}$.

TABLE II

The values of $\gamma_{i}^{\mathrm{L}}$.

\begin{tabular}{c|c|c|c}
\hline \hline & $\gamma_{1}^{\mathrm{L}}$ & $\gamma_{2}^{\mathrm{L}}$ & $\gamma_{3}^{\mathrm{L}}$ \\
\hline InAs & 19.67 & 8.37 & 9.29 \\
GaAs & 6.85 & 2.1 & 2.9
\end{tabular}

Then we define $H_{\mathrm{s}}$, which could bring the strain variations by relating to other elements

$$
H_{\mathrm{s}}=\left(\begin{array}{cccccccc}
a_{\mathrm{c}} e & 0 & -v^{\dagger} & 0 & -\sqrt{3} v & \sqrt{2} u & u & -\sqrt{2} v^{\dagger} \\
0 & a_{\mathrm{c}} e & \sqrt{2} u & \sqrt{3} v^{\dagger} & 0 & v & -\sqrt{2} v & -u \\
-v & \sqrt{2} u & -p+q & -s^{\dagger} & r & 0 & \sqrt{\frac{3}{2}} s & -\sqrt{2} q \\
0 & \sqrt{3} v & -s & -p-q & 0 & r & -\sqrt{2} r & \sqrt{\frac{1}{2}} s \\
-\sqrt{3} v^{\dagger} & 0 & r^{\dagger} & 0 & -p-q & s^{\dagger} & \sqrt{\frac{1}{2}} s^{\dagger} & \sqrt{2} r^{\dagger} \\
\sqrt{2} u & v^{\dagger} & 0 & r^{\dagger} & s & -p+q & \sqrt{2} q & \sqrt{\frac{3}{2}} s^{\dagger} \\
u & -\sqrt{2} v^{\dagger} & \sqrt{\frac{3}{2}} s^{\dagger} & -\sqrt{2} r^{\dagger} & \sqrt{\frac{1}{2}} s & \sqrt{2} q & -a_{\mathrm{v}} e & 0 \\
-\sqrt{2} v & -u & -\sqrt{2} q & \sqrt{\frac{1}{2}} s^{\dagger} & \sqrt{2} r & \sqrt{\frac{3}{2}} s & 0 & -a_{\mathrm{v}} e
\end{array}\right) .
$$

Here the components of $H_{\mathrm{s}}$ are given

$$
\left\{\begin{array}{l}
p=a_{\mathrm{v}}\left(e_{x x}+e_{y y}+e_{z z}\right)=a_{\mathrm{v}} e \\
q=b\left[e_{z z}-\frac{1}{2}\left(e_{x x}+e_{y y}\right)\right]= \\
\quad \frac{1}{2} b\left[2 e_{z z}-\left(e_{x x}+e_{y y}\right)\right] \\
r=\frac{\sqrt{3}}{2} b\left(e_{x x}-e_{y y}\right)-\mathrm{i} d e_{x y} \\
s=-d\left(e_{x z}-\mathrm{i} e_{y z}\right), \\
u=\frac{1}{\sqrt{3}} p_{0}\left(e_{z x} k_{x}+e_{z y} k_{y}+e_{z z} k_{z}\right), \\
v=\frac{1}{\sqrt{6}} p_{0}\left[\left(e_{x x}-\mathrm{i} e_{y x}\right) k_{x}+\left(e_{x y}-\mathrm{i} e_{y y}\right) k_{y}\right. \\
\left.\quad+\left(e_{x z}-\mathrm{i} e_{y z}\right) k_{z}\right] .
\end{array}\right.
$$

$a_{\mathrm{c}}$ denotes the conduction band deformation potential, $a_{\mathrm{v}}$ denotes the hydrostatic valence-band deformation, $e_{i j}$ denotes the strain tensor, and the shear deformation potentials are $b$ and $d$. The third part of $H$ is $V$, which is expressed as

$$
V=\left[\begin{array}{cccccccc}
E_{\mathrm{c}} & 0 & 0 & 0 & 0 & 0 & 0 & 0 \\
0 & E_{\mathrm{c}} & 0 & 0 & 0 & 0 & 0 & 0 \\
0 & 0 & E_{\mathrm{v}} & 0 & 0 & 0 & 0 & 0 \\
0 & 0 & 0 & E_{\mathrm{v}} & 0 & 0 & 0 & 0 \\
0 & 0 & 0 & 0 & E_{\mathrm{v}} & 0 & 0 & 0 \\
0 & 0 & 0 & 0 & 0 & E_{\mathrm{v}} & 0 & 0 \\
0 & 0 & 0 & 0 & 0 & 0 & E_{\mathrm{v}} & 0 \\
0 & 0 & 0 & 0 & 0 & 0 & 0 & E_{\mathrm{v}}
\end{array}\right] .
$$

When using the COMSOL software to complete the numerical calculations, we should do some necessary work in advance. Due to the fact that many parameters mentioned above cannot be substituted into the formulae in COMSOL directly, we need some changes. The following formulae derivations and transformations will help us to simplify the calculations and improve efficiency, so they are very important. For convenience, set $\hbar^{2} / 2 m_{0}=c c$, then the components of $H_{0}$ can be adapted as follows: 


$$
\begin{aligned}
& A_{\mathrm{c}}=\left[\begin{array}{ccc}
c c & 0 & 0 \\
0 & c c & 0 \\
0 & 0 & c c
\end{array}\right]=c c \cdot I, \\
& P_{\mathrm{c}}=\gamma_{1} c c \cdot I \text {, } \\
& Q_{\mathrm{c}}=\left[\begin{array}{ccc}
\gamma_{2} c c & 0 & 0 \\
0 & \gamma_{2} c c & 0 \\
0 & 0 & -2 \gamma_{2} c c
\end{array}\right] \text {, } \\
& P_{\mathrm{c}}+Q_{\mathrm{c}}=\left[\begin{array}{ccc}
\left(\gamma_{1}+\gamma_{2}\right) c c & 0 & 0 \\
0 & \left(\gamma_{1}+\gamma_{2}\right) c c & 0 \\
0 & 0 & \left(\gamma_{1}-2 \gamma_{2}\right) c c
\end{array}\right] \text {, } \\
& P_{\mathrm{c}}-Q_{\mathrm{c}}=\left[\begin{array}{ccc}
\left(\gamma_{1}-\gamma_{2}\right) c c & 0 & 0 \\
0 & \left(\gamma_{1}-\gamma_{2}\right) c c & 0 \\
0 & 0 & \left(\gamma_{1}+2 \gamma_{2}\right) c c
\end{array}\right] \text {, } \\
& -(P+Q)_{\mathrm{c}}=-c c\left[\begin{array}{ccc}
\gamma_{1}+\gamma_{2} & 0 & 0 \\
0 & \gamma_{1}+\gamma_{2} & 0 \\
0 & 0 & \gamma_{1}-2 \gamma_{2}
\end{array}\right] \text {, } \\
& -(P-Q)_{c}=-c c\left[\begin{array}{ccc}
\gamma_{1}-\gamma_{2} & 0 & 0 \\
0 & \gamma_{1}-\gamma_{2} & 0 \\
0 & 0 & \gamma_{1}+2 \gamma_{2}
\end{array}\right] \text {, } \\
& R_{c c}=\left[\begin{array}{ccc}
-\sqrt{3} c c \cdot \gamma_{2} & \mathrm{i} 2 \sqrt{3} c c \cdot \gamma_{3} & 0 \\
0 & \sqrt{3} c c \cdot \gamma_{2} & 0 \\
0 & 0 & 0
\end{array}\right]= \\
& -c c\left[\begin{array}{ccc}
\sqrt{3} \gamma_{2} & -\mathrm{i} \sqrt{3} \gamma_{3} & 0 \\
-\mathrm{i} \sqrt{3} \gamma_{3} & -\sqrt{3} \gamma_{2} & 0 \\
0 & 0 & 0
\end{array}\right] \\
& S_{c c}=2 \sqrt{3} c c \cdot \gamma_{3}\left[\begin{array}{ccc}
0 & 0 & 1 \\
0 & 0 & -\mathrm{i} \\
0 & 0 & 0
\end{array}\right]=
\end{aligned}
$$

Then

$$
\begin{aligned}
& U_{\text {beta }} / u_{\text {beta }}=\frac{-\mathrm{i} P_{0}}{\sqrt{3}}\left[\begin{array}{c}
-e_{z x} \\
-e_{z y} \\
1-e_{z z}
\end{array}\right]=\frac{\mathrm{i} P_{0}}{\sqrt{3}}\left[\begin{array}{c}
e_{z x} \\
e_{z y} \\
e_{z z}-1
\end{array}\right], \\
& V_{\text {beta }} / v_{\text {beta }}=\frac{-\mathrm{i} P_{0}}{\sqrt{6}}\left[\begin{array}{c}
1 /\left(e_{x x}-\mathrm{i} e_{y x}\right) \\
-\mathrm{i} /\left(e_{x y}-\mathrm{i} e_{y y}\right) \\
-\left(e_{x z}-\mathrm{i} e_{y z}\right)
\end{array}\right]= \\
& \frac{-P_{0}}{\sqrt{6}}\left[\begin{array}{c}
\mathrm{i}-\left(\mathrm{i} e_{x x}+e_{y x}\right) \\
1-\left(\mathrm{i} e_{x y}+e_{y y}\right) \\
-\left(e_{y z}+\mathrm{i} e_{x z}\right)
\end{array}\right]
\end{aligned}
$$

Substitute these components above in formula to solve equation as follows:

$$
\nabla(-c \nabla u)+\beta \nabla u+d u=\lambda u .
$$

Meanwhile we give most of material parameters used for calculations in the paper, as Table III.

The material parameters of InAs/GaAs used for calculations.

\begin{tabular}{l|c|c|c|c|c|c|c|c|c|c|c}
\hline \hline & $m_{0}$ & $\varepsilon_{r}$ & $C_{11}$ & $C_{12}$ & $C_{44}$ & $E_{\mathrm{c}}$ & $E_{\mathrm{v}}$ & $a_{\mathrm{c}}$ & $a_{\mathrm{v}}$ & $e_{14}$ & $b$ \\
\hline InAs & 0.04 & 15.2 & 83.4 & 45.4 & 39.5 & 0.67 & 0.25 & -5.08 & 1 & 0.045 & -1.8 \\
GaAs & 0.0667 & 12.9 & 118.8 & 53.8 & 59.4 & 1.519 & 0 & -7.17 & 1.16 & 0.16 & -1.7
\end{tabular}

\section{Results and discussions}

Consider the InAs/GaAs QD as a square-based pyramid. Although the wetting layer does not influence the strain distributions obviously, we still introduce it for completeness of the true model. The thickness of wetting layer is $2 \mathrm{~nm}$. The growth direction is along the (001) direction. The thickness of cap and substrate are $10 \mathrm{~nm}$ and $40 \mathrm{~nm}$, respectively. The height of the quantum dot is $6 \mathrm{~nm}$. The length of the base is $12 \mathrm{~nm}$, as in Fig. 1. 


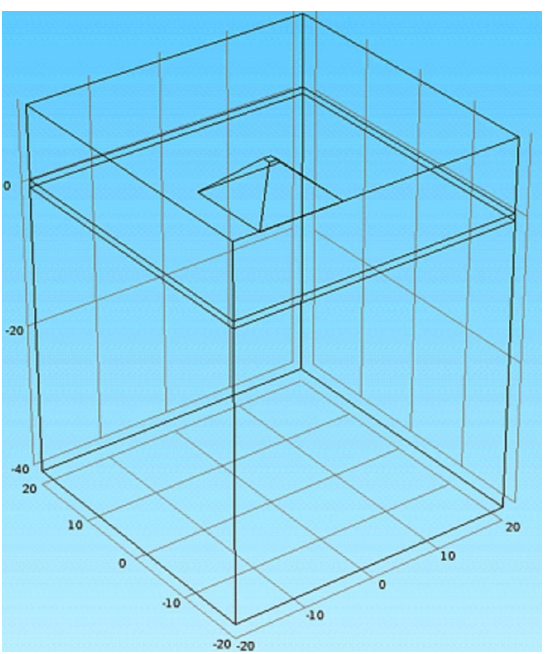

Fig. 1. Schematic drawing of the geometry of QD.

When we observe the strain distributions along a straight line through the center of the QD with the (001) direction, the results are shown in Fig. 2, where $\varepsilon_{x x}, \varepsilon_{y y}$, $\varepsilon_{z z}$, hydrostatic $\left(\varepsilon_{\mathrm{h}}\right)$ and bi-axial $\left(\varepsilon_{\mathrm{b}}\right)$ strain distributions are corresponding to Fig. $2 \mathrm{a}-\mathrm{e}$, respectively.

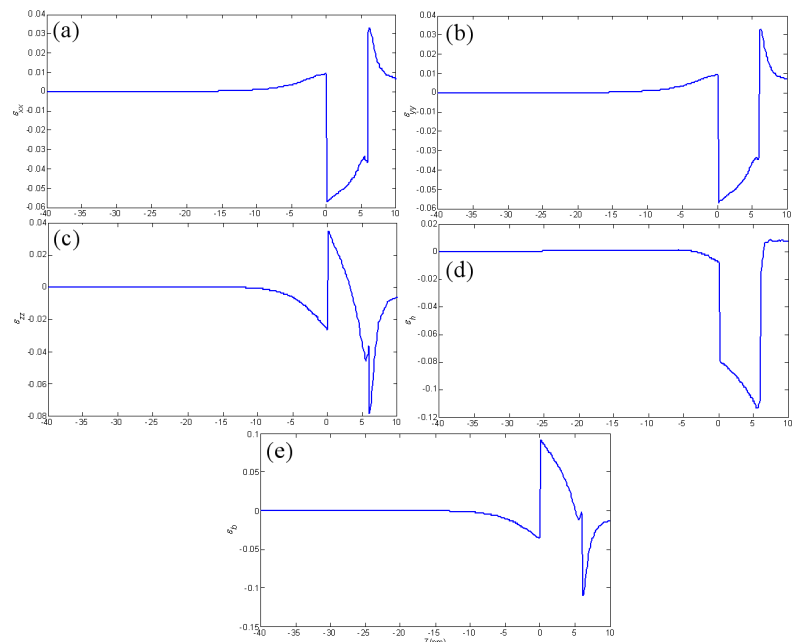

Fig. 2. The strain distributions along the (001) direction through the centre of the QD. (a)-(c) are the $\varepsilon_{x x}$, $\varepsilon_{y y}, \varepsilon_{z z}$ strain components, respectively. (d), (e) are the hydrostatic and bi-axial strain distributions, respectively.

The strain distributions along the cross-sections of the (010) plane through the center of the QD and in threedimensional space are given in Fig. 3 and Fig. 4, where $\varepsilon_{x x}, \varepsilon_{y y}, \varepsilon_{z z}, \varepsilon_{\mathrm{h}}$, and $\varepsilon_{\mathrm{b}}$ are corresponding to Fig. 3 and Fig. $4 \mathrm{a}-\mathrm{e}$, respectively.

Figure 2 presents that the strain value in the $\mathrm{QD}$ region produces a considerable change. The values of $\varepsilon_{x x}$ and $\varepsilon_{y y}$ are equal along the (001) direction and manifest as compressive strain in the inner part of QD. In the region from the bottom to the top of $\mathrm{QD}$, the values of $\varepsilon_{x x}$

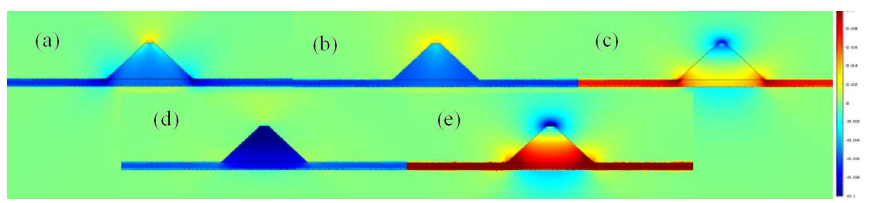

Fig. 3. The strain distributions along the cross sections of the (010) plane through the center of the QD. (a)-(c) are $\varepsilon_{x x}, \varepsilon_{y y}, \varepsilon_{z z}$ strain components, respectively. (d), (e) are the hydrostatic and bi-axial strain distributions, respectively.

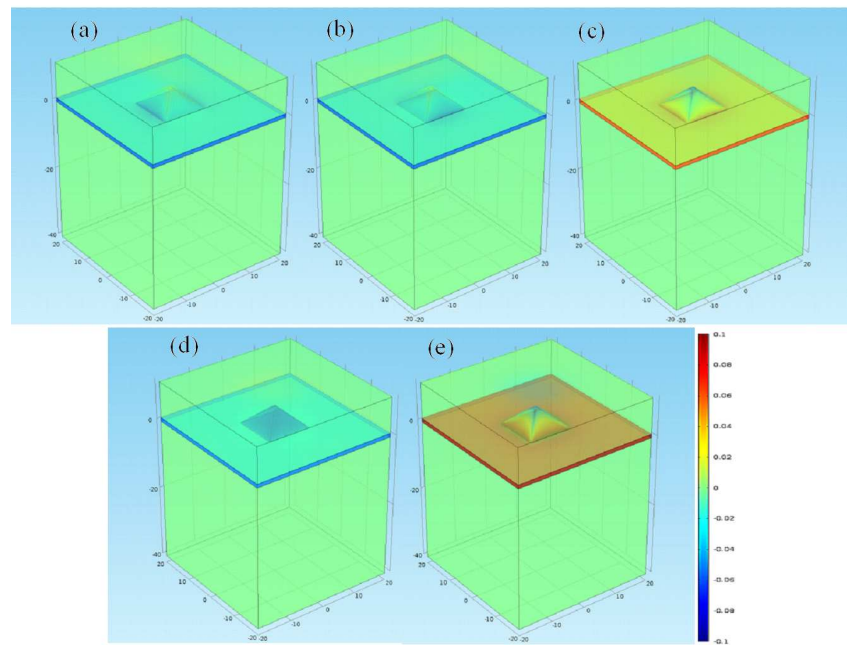

Fig. 4. The strain distributions of QD in threedimensional space. (a)-(c) are the $\varepsilon_{x x}, \varepsilon_{y y}, \varepsilon_{z z}$ strain components, respectively. (d), (e) are the hydrostatic and bi-axial strain distributions, respectively.

and $\varepsilon_{y y}$ increase slowly. It means the relaxation has happened in the QD but not enough. Near the bottom of QD, the coherency phenomenon of InAs and GaAs lattices is obvious, leading to a large strain. Near the top of QD, the strain is smaller due to fact that lots of strain are released during the process of quantum dot growth. In the region outside the $\mathrm{QD}$, the value of $\varepsilon_{x x}$ is greater than zero which is consistent with the experimental results.

Next, we discuss the hydrostatic strain and the bi-axial strain. The hydrostatic strain will cause the movement of band edges while the bi-axial strain will cause the separation between heavy hole band and light hole band. Both two strains play a decisive role in terms of electronic potential, discreteness of light waves and the state of electron holes and also affect the study of quantum dot devices. Figure 2 presents the hydrostatic strain distribution in the QD. The value of $\varepsilon_{\mathrm{h}}$ is very small in the substrate and cap layer that is generally considered as zero while $\varepsilon_{\mathrm{h}}$ manifest as compressive strain in the inner part of QD. We can think of the hydrostatic strain in the QD as a potential well. Furthermore, a finite depth potential well is formed in the (001) direction of QD benefiting from the hydrostatic strain. Note that the hydrostatic strain is not affected by the $Q$ value but the biaxial strain is. The value of $\varepsilon_{\mathrm{b}}$ is positive near the base of $\mathrm{QD}$ and negative near the top which means with the 
change of symbol, the energy level of heavy holes begin to be lower than the light holes. The bigger $Q$ value, the change taking place is closer to the top of QD.

Form Fig. 3 and Fig. 4 one can notice that, to the $\varepsilon_{x x}$ and $\varepsilon_{y y}$ strain component, the compressive strain mainly distribute in the inner part of $\mathrm{QD}$ and wetting layer and the main concentration of tensile strain is at the top of QD. To $\varepsilon_{z z}$ strain component, the compressive strain mainly distribute at the top and the tensile strain mainly distribute in the inner part of $\mathrm{QD}$ and wetting layer. The hydrostatic strain also distribute in the inner part of QD and wetting layer, the value is negative, and in other regions approximates to zero. The bi-axial strain in the inner part of QD and wetting layer manifests as tensile strain while compressive strain - at the top of QD. These conclusions are consistent with the observations for one-dimensional case.

We know that the shear strain components can decide the strength of the linear polarizations, however, it is not enough when we discuss the second order polarizations, the normal strains should be considered. Figure 5 shows the 3D piezoelectric potential distribution.

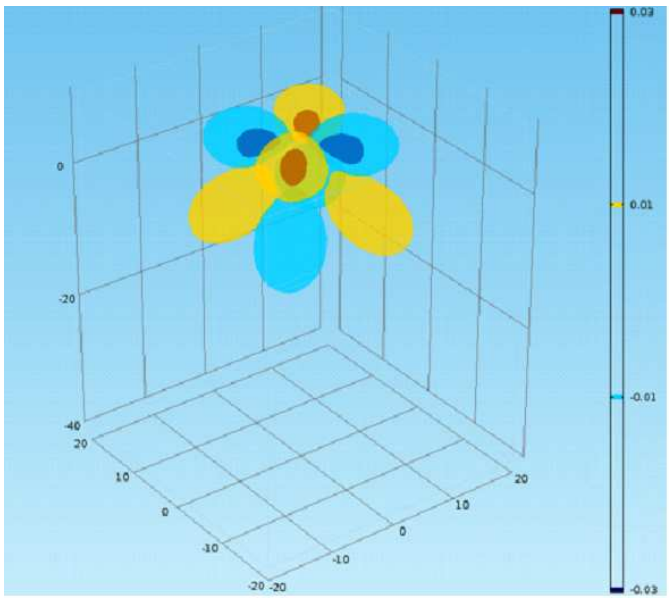

Fig. 5. 3D piezoelectric potential distribution.

From Fig. 5 one can notice that the total piezoelectric potential presents a $C_{2 v}$ symmetry, different with the symmetry of QD $\left(C_{4 v}\right)$, which results in the exciton fine structure splitting of QD. Furthermore, if the InAs/GaAs QD grows along the (111) direction, the piezoelectric potential will present a $C_{3 v}$ symmetry. By this time, the electronic confining potential still has a good symmetry to restrict the exciton fine structure splitting $[16,17]$.

The electronic structures are also given in Fig. 6.

By solving Eq. (18) numerically, we can obtain the bound states as shown in Fig. 6. As we can see, the wave functions are symmetrically distributed in the QD meanwhile electrons and holes are well confined in the QD.

Finally we give some calculation settings and hardware configuration as references: the meshes of substrate, cap layer, wetting layer and inner part of $\mathrm{QD}$ are $3,3,2$, and $1 \mathrm{~nm}$, respectively. A computer with 16 cores (i7)

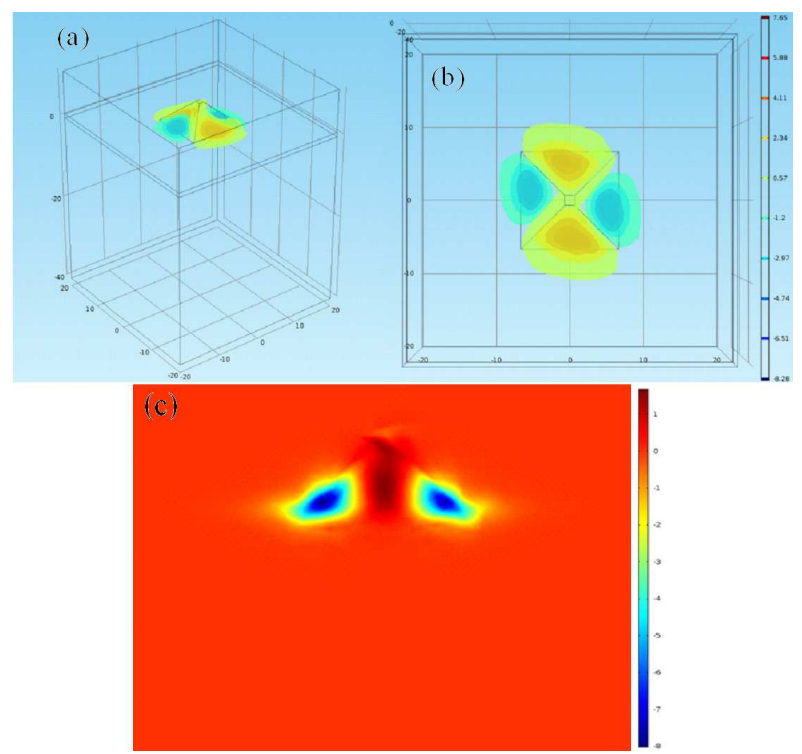

Fig. 6. The electron probability density distributions. (a), (b) and (c) are 3D stereogram, overhead view and sectional view, respectively.

and 128 G memory is used. The software version of COMSOL is $4.3 \mathrm{~b}$. A complete calculation will cause about $44 \mathrm{~min} 50 \mathrm{~s}$, perfectly acceptable for most of researchers.

\section{Conclusions}

The strain distributions, piezoelectric effects, and the electronic structure of pyramidal InAs/GaAs QD are calculated in this paper. The theoretical bases of calculation are the continuum elastic theory, eight band $k \cdot p$ theory and the finite element method. Compared to the traditional method, we use the COMSOL software to solve the eight band $k \cdot p$ Hamiltonian directly. Some derivations and transformations about the parameters which are used in calculation are given. The calculated results show that both strain distributions and piezoelectric effects have a significant impact on the electronic structure of InAs/GaAs QD, verify the feasibility and efficiency of the calculation method. The whole solving process will have a reference value to other researchers.

\section{Acknowledgments}

The author acknowledges the funding of following science foundations: National Natural Science Foundation of China (grant Nos. 60908028, 60971068, 10979065, and 61275201).

\section{References}

[1] A. Gonzalez, B. Partoens, F.M. Peeters, Phys. Rev. B 56, 15740 (1997).

[2] R. Heitz, A. Kalburge, Q. Xie, Phys. Rev. B 57, 9050 (1998).

[3] C. Bose, C. Chakraborty, C.K. Sarkar, Solid-State Electron. 41, 1383 (1997).

[4] I.P. Radko, M.G. Nielsen, O. Albrektsen, Opt. Expr. 18, 18633 (2010). 
[5] W.B. Yu, A. Madhukar, Phys. Rev. Lett. 79, 905 (1997).

[6] A.D. Andreev, E.P. O'Reilly, Phys. Rev. B 62, 15851 (2000).

[7] A.V. Dvurechenskii, A.V. Nenashev, A.I. Yakimov, Nanotechnology 13, 75 (2002).

[8] Y. Kikuchi, H. Sugii, K. Shintani, J. Appl. Phys. 89, 1191 (2001)

[9] G.S. Pearson, D.A. Faux, J. Appl. Phys. 88, 730 (2000).

[10] T. Benabbas, Y. Androussi, J. Appl. Phys. 86, 1945 (1999).

[11] C. Pryor, Phys. Rev. B 57, 7190 (1998).
[12] A. Schliwa, M. Winkelnkemper, D. Bimberg, Phys. Rev. B 76, 205324 (2007).

[13] M.A. Migliorato, D. Powell, A.G. Cullis, Phys. Rev. B 74, 245332 (2006).

[14] G. Bester, X.F. Wu, D. Vanderbilt, Phys. Rev. Lett. 96, 187602 (2006).

[15] G. Bester, A. Zunger, X.F. Wu, Phys. Rev. B 74, 081305 (2006).

[16] A. Schliwa, M. Winkelnkemper, A. Lochmann, Phys. Rev. B 80, 161307(R) (2009).

[17] R. Singh, G. Bester, Phys. Rev. Lett. 103, 063601 (2009). 